EPJ Web of Conferences 75, 07003 (2014)

DOI: $10.1051 /$ epjconf/ 20147507003

(C) Owned by the authors, published by EDP Sciences, 2014

\title{
Substitutional effects of In by Cu in Celn
}

\author{
D. P. Rojas ${ }^{1}$, J. I. Espeso² ${ }^{2}$ and J Rodríguez Fernández ${ }^{2, a}$ \\ ${ }^{1}$ Departamento de Física e Instalaciones-ETSAM, Universidad Politécnica de Madrid, Av. Juan Herrera, 4. 28040, Madrid. Spain \\ ${ }^{2}$ CITIMAC \& MAGMA, UA-CSIC, Facultad de Ciencias, Universidad de Cantabria, Av. de los Castros s/n.39005, Santander. \\ Spain
}

\begin{abstract}
We have investigated the evolution of the magnetic properties on the $\mathrm{Ce}\left(\operatorname{In}_{1-x} \mathrm{Cu}_{x}\right)_{2}(0<x \leq 0.3)$ series of alloys. The orthorhombic structure of the $\mathrm{CeIn}_{2}$ alloy (Imma) changes into the hexagonal $\mathrm{AlB}_{2}$-type $(P 6 / \mathrm{mmm})$ for $x=0.05$ and, then, into the hexagonal $\mathrm{CaIn}_{2}$-type $\left(\mathrm{PC}_{3} / \mathrm{mmm}\right)$ for higher $\mathrm{Cu}$ concentrations, up to $x=0.3$. The $\mathrm{dc}$ (ac) magnetic susceptibility shows an abrupt decrease of the magnetic transition temperature from $22 \mathrm{~K}$ to $5.4 \mathrm{~K}(x=0.05)$. The results indicate the influence of the crystallographic type of structure and disorder effects on the magnetic behavior along the series.
\end{abstract}

\section{Introduction}

Ce intermetallic compounds have constituted a subject of intensive studies because of their interesting properties [1]. In the study of this kind of materials, the chemical substitution has been commonly used to tune the different physical properties, despite it may introduce disorder in the given system. For instance, an evolution from a Pauli paramagnet $(\mathrm{CeNi})$ to a Kondo ferromagnet $(\mathrm{CePt})$, with a crossover around $x=0.90$ has been found in the study of the $\mathrm{CeNi}_{x} \mathrm{Pt}_{1-x}$ series of alloys [2]. Sometimes, even the introduced disorder itself is at the origin of novel and relevant phenomena, such as the emergence of mesoscopic magnetic states due to the clustering of disordered magnetic moments [3].

In this context, the nature of the first-order ferromagnetic (FM) transition $\left(T_{C}=22 \mathrm{~K}\right)$ of the $\mathrm{CeIn}_{2}$ alloy [4] has been studied by combining chemical substitution with muon spin rotation spectroscopy $(\mu \mathrm{SR})$ experiments, which unveiled the existence of an intermediate antiferromagnetic phase around $23 \mathrm{~K}$ [5].

In the present work, we undertake a study of the chemical substitution effects of In by $\mathrm{Cu}$ on the magnetic properties of the $\mathrm{CeIn}_{2}$ alloy. The evolution of the magnetic behavior from first-order FM in CeIn ${ }_{2}$ to spin-glass (SG) in $\mathrm{Ce}_{2} \mathrm{CuIn}_{3}[6,7]$ (which corresponds to $x=0.25$ in the $\mathrm{Ce}\left(\operatorname{In}_{1-x}(\mathrm{Cu})_{x}\right)_{2}$ series of alloys) will be monitored by measurements of the magnetic susceptibility (both ac and $\mathrm{dc}$ ) for different $\mathrm{Cu}$ concentrations on the series of $\mathrm{Ce}\left(\operatorname{In}_{1-x}(\mathrm{Cu})_{x}\right)_{2}$ alloys. The results will be compared with those obtained from the chemical substitution of In by $\mathrm{Ni}$ in the $\mathrm{CeIn}_{2}$ alloy [8].

\footnotetext{
ae-mail: rodrigufj@unican.es
}

\section{Experimental}

Different compositions from the series $\mathrm{Ce}\left(\mathrm{In}_{1-x} \mathrm{Cu}_{x}\right)_{2}(x$ $=0.05,0.15,0.2$, and 0.3 ) were prepared from the constituent materials $\mathrm{Ce}$ (4N-Alfa), In (4N-Alfa) and $\mathrm{Cu}(5 \mathrm{~N}-$ Alfa) in an arc-melting furnace, under a protective $\mathrm{Ar}$ atmosphere. Systematic X-ray diffraction patterns were acquired using $\mathrm{CuK}_{\alpha}$ radiation in a Philips diffractometer. The $\mathrm{dc}(\mathrm{ac})$-magnetic susceptibility measurements were carried out in a Quantum Design PPMS device.

\section{Results and discussion}

\subsection{Structural characterization}

The X-ray powder diffraction experimental data on $\mathrm{Ce}\left[\operatorname{In}_{1-x}(\mathrm{Cu})_{x}\right]_{2}$ alloys show that the orthorhombic $\mathrm{CeCu} u_{2}$-type structure (Imma space group), found for the binary $\mathrm{CeIn}_{2}$ alloy, changes, for a small $\mathrm{Cu}$ substitution $(x=0.05)$, into an hexagonal $A l B_{2}$-type one $(P 6 / \mathrm{mmm}$ space group). This change of structure implies a strong modification of the local coordination of both the $\mathrm{Ce}$ ion and the transition metals ( $\mathrm{In}, \mathrm{Cu}$ ), randomly distributed in the same crystallographic site.

For higher concentrations $(x=0.15,0.2,0.3)$, the planar hexagonal coordination of the transition metals along the basal plane gets distorted with a zigzag along the $c$ axis and, then, the crystallographic structure becomes hexagonal $\mathrm{CaIn}_{2}$-type ( $P 6_{3} / \mathrm{mmc}$ space group), as derived from the Rietveld refinement of the X-ray data. As an example, the refinement corresponding to the concentration $x=0.2$ is presented in figure 1 . Small traces, less than $2 \%$, of the $\mathrm{CeIn}_{3}$ phase were also found only in the sample with $x=0.05$. However, we should remark that, despite the $\mathrm{CeIn}_{3}$ alloy is known to antiferromagnetically order at 10 $\mathrm{K}[9]$ (temperature far away from the ordering ones of the 
Table 1. Values of the lattice parameters for different samples in the $\mathrm{Ce}\left(\mathrm{In}_{1-x} \mathrm{Cu}_{x}\right)_{2}(0<x \leq 0.3)$ series of alloys. ${ }^{a}$ Value extracted from the literature [7].

\begin{tabular}{cccc}
\hline conc. $(\mathrm{x})$ & $\mathrm{a}(\AA)$ & $\mathrm{b}(\AA)$ & $\mathrm{c}(\AA)$ \\
\hline 0.05 & $4.909(4)$ & $4.909(4)$ & $3.849(5)$ \\
0.15 & $4.869(4)$ & $4.869(4)$ & $7.661(1)$ \\
0.20 & $4.842(3)$ & $4.842(3)$ & $7.644(1)$ \\
$0.25^{a}$ & $4.822(1)$ & $4.822(1)$ & $7.680(1)$ \\
0.30 & $4.801(6)$ & $4.801(6)$ & $7.679(1)$ \\
\hline
\end{tabular}

samples studied in the present work), no evidence of any magnetic transition at $10 \mathrm{~K}$ has been observed in any of the magnetic measurements performed on our samples. The analysis of the X-ray diffraction patterns yields the lattice parameters presented in table 1 . The values for $x=0.25$ were taken from the previous literature [7].

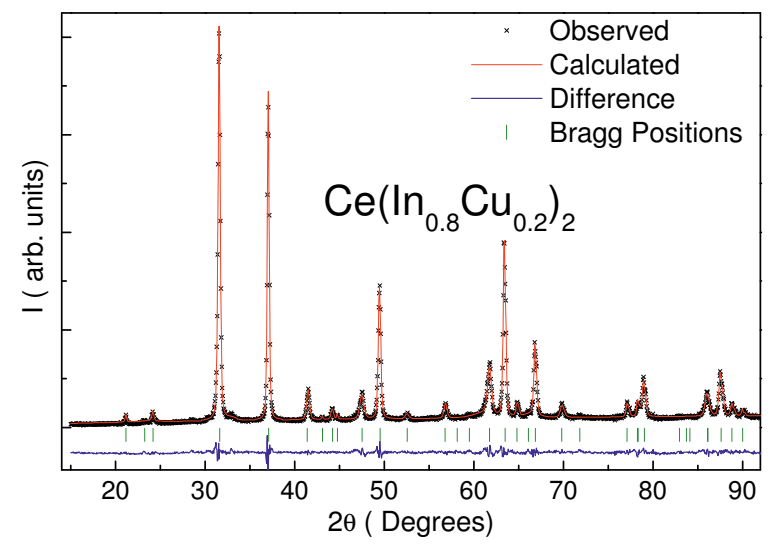

Figure 1. X-ray diffraction pattern of the $\mathrm{Ce}\left(\mathrm{In}_{0.8} \mathrm{Cu}_{0.2}\right)_{2}$ alloy). The solid line is the result of the Rietveld refinement and the experimental-calculated difference is depicted below. The vertical markers indicate the Bragg positions according to the hexagonal $\mathrm{PG}_{3} / \mathrm{mmc}$ crystallographic structure.

\section{$3.2 \mathrm{dc}$ and ac-magnetic susceptibility}

The results of the dc-magnetic susceptibility $(\chi=\mathrm{M} / \mathrm{H})$ measurements at different magnetic fields for the $\mathrm{Ce}\left(\mathrm{In}_{0.95} \mathrm{Cu}_{0.05}\right)_{2}$ alloy are shown in figure 2 . The first fact that we can notice is that a small chemical substitution, of just $5 \%$ of $\mathrm{Cu}$ into the In crystallographic site, strongly influences the ferromagnetic transition temperature. In fact, there is an abrupt decrease, from $22 \mathrm{~K}$ in $\mathrm{CeIn}_{2}$ to $5.1 \mathrm{~K}$ in the sample with $x=0.05$. Furthermore, the zero field cooled (ZFC) and field cooled (FC) curves show a relatively large irreversibility at low magnetic field (100 Oe), which diminishes when increasing the magnetic field up to 500 Oe.

Increasing the $\mathrm{Cu}$ concentration up to $x=0.15$ $\left(\mathrm{Ce}\left(\operatorname{In}_{0.85} \mathrm{Cu}_{0.15}\right)_{2}\right.$ alloy), the ferromagnetic transition slightly shifts to lower temperatures, down to $4.0 \mathrm{~K}$, as shown in figure 3 . From a qualitative point of view, the

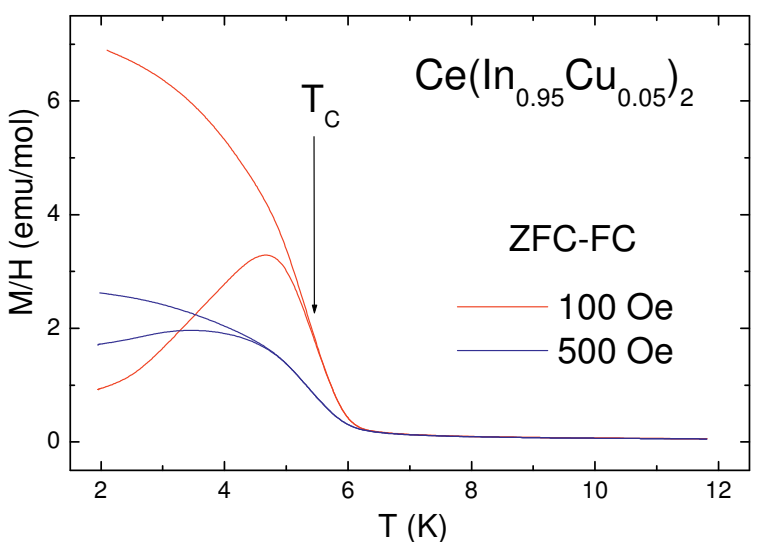

Figure 2. ZFC and FC curves of dc-magnetic susceptibility at different magnetic fields for the $\mathrm{Ce}\left(\mathrm{In}_{0.95} \mathrm{Cu}_{0.05}\right)_{2}$ alloy. The ferromagnetic transition is indicated by a marker.

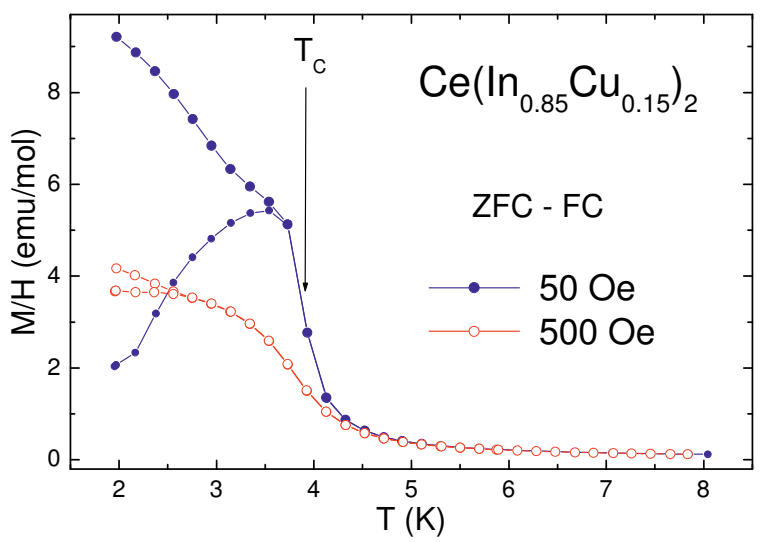

Figure 3. Temperature dependence of ZFC and FC curves of the dc-magnetic susceptibility for the $\mathrm{Ce}\left(\mathrm{In}_{0.85} \mathrm{Cu}_{0.15}\right)_{2}$ alloy at $50 \mathrm{Oe}$ and 500 Oe.

general behavior of this magnetic susceptibility is similar to the one observed in the previous composition $(x=0.05)$; i.e, there is a large irreversibility at low magnetic fields that almost disappears when increasing the magnetic field up to 500 Oe. This irreversibility can arise from the dynamics of the ferromagnetic domains or from some kind of disordered magnetic arrangement. That is why we have further investigated this sample by ac-magnetic susceptibility and these results are presented in figure 4. A frequencyindependent peak, associated to the ferromagnetic transition, appears around $4 \mathrm{~K}$. As the transition temperature does not depend on the excitation frequency, we can discard the existence of a spin-glass-like magnetic ground state, therefore confirming the FM behavior of this sample. The ac-susceptibility measurements for the sample with smaller $\mathrm{Cu}$ concentration $(x=0.05)$ show a similar behavior, with a magnetic transition temperature at $5.4 \mathrm{~K}$. Both Curie temperature values are in agreement with those other obtained from the dc-susceptibility measurements. 


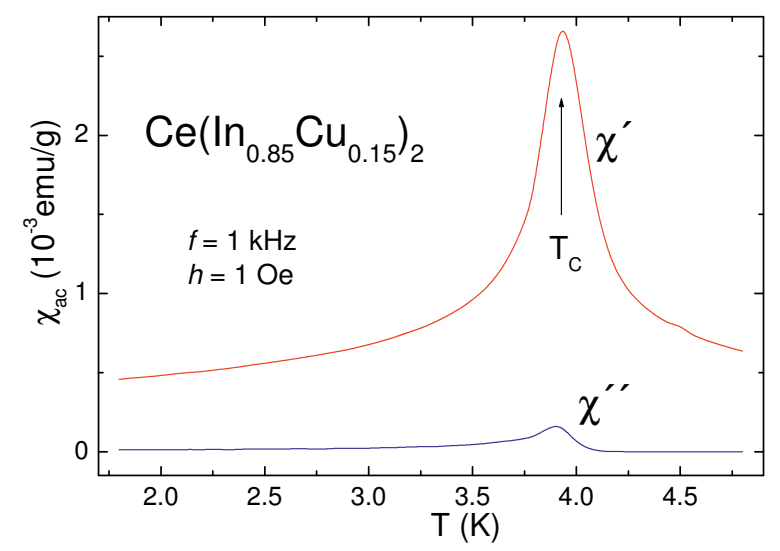

Figure 4. Temperature dependence of the real $\left(\chi^{\prime}\right)$ and imaginary $\left(\chi^{\prime \prime}\right)$ components of the ac-magnetic susceptibility for the sample with $x=0.15$ at a frequency of $1 \mathrm{kHz}$. A peak at $T_{C}$ is observed in both components.

A change in the magnetic behavior takes place when the $\mathrm{Cu}$ concentration is increased above $x=0.15$. The best way to evaluate this change is to track the results of the ac-magnetic susceptibility, as shown in figure 5 for the $\mathrm{Ce}\left(\mathrm{In}_{0.8} \mathrm{Cu}_{0.2}\right)_{2}$ alloy $(x=0.2)$. In fact, the peaks observed in both $\chi^{\prime}$ and $\chi^{\prime \prime}$ shift to higher temperatures when the frequency is increased. This feature is characteristic of a spin-glass (SG) behavior. In addition, the value of the relative shift per frequency decade, $\Delta T_{f} / T_{f} \Delta \log v=0.0227$, is similar to those reported for other Non-Magnetic Atom Disorder SG, such as $\mathrm{Ce}_{2} \mathrm{CuIn}_{3}$ [6], $\mathrm{CeNi}_{1-x} \mathrm{Cu}_{x}$ [10], or $\mathrm{Ce}_{2} \mathrm{AgIn}_{3}$ [11]. It is worth to mention that the first one of these systems corresponds to a $\mathrm{Cu}$ concentration $x=0.25$ in the $\mathrm{Ce}\left(\operatorname{In}_{1-x} \mathrm{Cu}_{x}\right)_{2}$ series of alloys. This composition presents a freezing temperature $T_{f}=2.1 \mathrm{~K}$, as determined from the maximum of the ac-susceptibility at the lowest measured frequency $(1 \mathrm{~Hz})$, which is close to that observed in the alloy with $x=0.2\left(T_{f}=2.2 \mathrm{~K}\right)$. On the other hand, the $\mathrm{Ce}\left(\mathrm{In}_{0.7} \mathrm{Cu}_{0.3}\right)_{2}$ alloy $(x=0.3)$ displays a similar behavior to the one exhibited by the samples with $x=0.2$ and 0.25 , but with slightly larger values of $T_{f}=2.65 \mathrm{~K}$ and $\Delta T_{f} / T_{f} \Delta \log v=0.0376$.

\section{Discussion}

From the results presented in the previous section, it is obvious that the chemical substitution strongly influences the magnetic properties of the $\mathrm{CeIn}_{2}$ alloy, when In is replaced by a transition metal such as $\mathrm{Cu}$. Notably, the phenomenology reported in this situation is different to the one reported for the $\mathrm{La}_{1-x} \mathrm{Ce}_{x} \mathrm{In}_{2}$ series of alloys. In that case, for La concentrations between $x=0$ and 0.1 , the FM transition shifts just a bit to lower temperatures (from 22 to $19.5 \mathrm{~K}$ ), and even the first-order character of the transition is kept. That decrease of the Curie temperature can even be directly attributed to the smaller concentration of magnetic atoms. On the other hand, we must also remind that the substitution of Ce by $\mathrm{La}$ in the $\mathrm{CeIn}_{2}$ alloy does

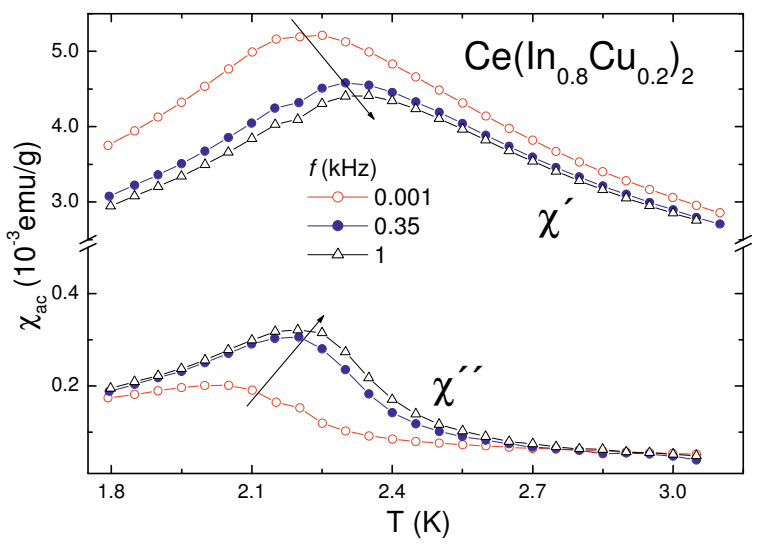

Figure 5. Temperature dependence of the real $\left(\chi^{\prime}\right)$ and imaginary $\chi^{\prime \prime}$ components of ac-magnetic susceptibility of $\mathrm{Ce}\left(\operatorname{In}_{0.8} \mathrm{Cu}_{0.2}\right)_{2}$. There is a shift of the peaks, in both components, to higher temperatures with the increase of the frequency.

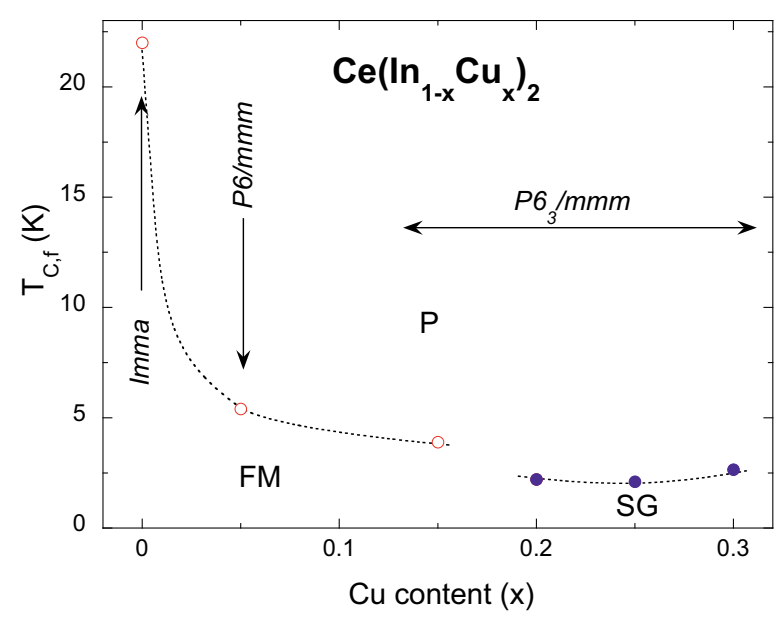

Figure 6. Phase diagram of the $\mathrm{Ce}\left(\operatorname{In}_{1-x} \mathrm{Cu}_{x}\right)_{2}$ series of alloys, reflecting the evolution of both the crystallographic structure and the magnetic behavior ( $\mathrm{P}$ stands for paramagnetism). A crossover from FM to SG state is found between $x=0.15$ and $x=0.2$. The value of $T_{f}$ for the sample with $x=0.25$ was taken from reference [7].

not affect the stability of the crystallographic orthorhombic structure, which remains the same in all the substituted samples [5].

However, in the $\mathrm{Ce}\left(\operatorname{In}_{1-x} \mathrm{Cu}_{x}\right)_{2}$ series, the crystallographic structure changes from orthorhombic (Imma for $x=0$ ) to hexagonal $P 6 / \mathrm{mmm}$ for the Cu-diluted sample with $x=0.05$. This crystallographic evolution is similar to that observed in the study of the $\mathrm{Ce}\left(\mathrm{In}_{1-x} \mathrm{Ni}_{x}\right)_{2}$ series [8]. Remarkably, in both cases, there is an abrupt decrease of the Curie temperature that can be directly associated with the change of the crystallographic structure. Moreover, in the $\mathrm{Cu}$-diluted series, there is an additional change of the crystallographic structure into the hexagonal $\mathrm{PG}_{3} / \mathrm{mmm}$ space group, for concentrations larger than $x=0.05$ (see Figure 6). As explained in section 3.1, this structure re- 
duces the symmetry of the crystallographic site in which the In and $\mathrm{Cu}$ ions are randomly distributed.

From the magnetic point of view, there is a crossover from FM to a SG behavior between the samples with composition $x=0.15$ and 0.20 . It is well known that the chemical disorder induced by the substitution of a transition metal into the In site, within both hexagonal structures reported in the present work, influences the magnetic interactions of the $\mathrm{Ce}$ ions, which results in a frustration of these interactions, and consequently, leading to a SG behavior, as observed in $\mathrm{Ce}_{2} \mathrm{NiIn}_{3}$ [6], $\mathrm{Ce}_{2} \mathrm{CuIn}_{3}$ [7] or $\mathrm{Ce}_{2} \mathrm{AgIn}_{3}$ [11]. Although there might be some influence of the crystallographic structure in the establishment of the SG ground state, these results, together with the ones presented in the actual work, seem to indicate that the chemical disorder plays the major role in the emergence of this disordered magnetic state.

It has also been shown that in disordered Ce alloys, the competition between ferromagnetism, SG and Kondo effect is crucial in defining the ground state and the magnetic behavior in these systems $[12,13]$. However, further experimental work would be necessary to evaluate the balance between these interactions.

\section{Conclusions}

The influence of the chemical substitution effects on the $\mathrm{CeIn}_{2}$ alloy, when $\mathrm{In}$ is replaced by $\mathrm{Cu}$, has been established by measurements of the magnetic properties in the $\mathrm{Ce}\left(\operatorname{In}_{1-x} \mathrm{Cu}_{x}\right)_{2}(0<x \leq 0.3)$ series of alloys. For a small amount of substituted In metal (5\%), a significant decrease on the Curie temperature is observed. This decrease can be associated with the change of crystallographic structure, from an orthorhombic Imma to an hexagonal P6/mmm one. Disorder effects seem to gain force with the increase of the $\mathrm{Cu}$ content, showing a crossover from FM to SG behavior for $x$ values between 0.15 and 0.2. In this case, the chemical disorder is mainly at the origin of the appearance of this SG behavior.
This work has been supported by the Direction of the Universities of the Ministry of Science and Education of Spain under contract MAT2011-27573-C04.

\section{References}

[1] J. Sereni, Handbook on the Physics and Chemistry of Rare Earths, vol. 15, ch.98 (Elsevier Science, Amsterdam, 1991)

[2] J. A. Blanco, M. de Podesta, J. I. Espeso, J. C. Gómez Sal, C. Lester, K. A. McEwen, N. Patrikios, J.Rodríguez Fernández, Phys Rev B 49, 15126 (1994)

[3] N. Marcano, J. C. Gómez Sal, J. I. Espeso, J. M. De Teresa, P. A. Algarabel, C. Paulsen, J. R. Iglesias, Phys. Rev. Lett. 98, 166406 (2007)

[4] D. P. Rojas, J. I. Espeso, J. Rodríguez Fernández, J. C. Gómez Sal, Phys. Rev B 80, 184413 (2009)

[5] D. P. Rojas, J. I. Espeso, J. Rodríguez Fernández, J. C. Gómez Sal, D. Andreica, C. Rusu, R. Dudric, A. Amato, Phys. Rev B 84, 024403 (2011)

[6] D. P. Rojas, L. C. J. Pereira, E. B. Lopes, J. C. Waerenborgh, L. M. da Silva, F. G. Gandra, A. N. Medina, J. Alloy Compd. 432, 34 (2007)

[7] D. P. Rojas, J. I. Espeso, J. Rodríguez Fernández, J. C. Gómez Sal, Physica B 378-380, 847 (2006)

[8] D. P. Rojas, J. I. Espeso, J. Rodríguez Fernández, J. Magn. Magn. Mater. 345, 190 (2013)

[9] J. M. Lawrence, S. M Shapiro, Phys Rev B 22, 4379 (1980)

[10] J. García Soldevilla, J. C. Gómez Sal, J. A. Blanco, J. I. Espeso, J. Rodríguez Fernández, Phys. Rev. B 61, 6821 (2000)

[11] T. Nishioka,Y. Tabata, T. Taniguchi, Y. Miyako, J. Phys. Soc. Jpn. 69, 1012 (2000)

[12] S. G. Magalhaes, F. M. Zimmer, P. R. Krebs, B. Coqblin, Phys. Rev. B 74, 014427 (2006)

[13] B. Coqblin, Acta Phys. Pol. A 118, 913 (2010) 